\title{
Neurotransmetteurs cérébraux et contrôle du comportement sexuel masculin
}

\author{
J. BuvAT
}

Association pour l'Etude de la pathologie de l'appareil reproducteur et de la psychosomatique (EPARP), 49 rue de la Bassée, 59000 Lille

\section{RESUME}

Des progrès importants, quoiqu'encore parcellaires, ont été accomplis dans la compréhension du rôle que jouent les neurotransmetteurs mono-aminergiques et peptidergiques dans le contrôle du comportement sexuel (CS) animal, et à un moindre degré humain. Parmi les mono-amines, la dopamine stimule tous les aspects du CS chez l'animal et au moins l'érection chez l'homme. La noradrénaline semble impliquée dans un tonus adrénergique central inhibiteur s'exprimant par l'intermédiaire des récepteurs alpha 2 . La sérotonine exerce des effets inhibiteurs ou stimulants selon le type de récepteur concerné, et peut particulièrement induire chez le rat un comportement de type éjaculation prématurée, alors que les inhibiteurs de sa recapture améliorent la dysfonction correspondante chez l'homme. Parmi les neuropeptides, les substances opioïdes et particulièrement la bétaendorphine inhibent tous les aspects du CS, et sont probablement des intermédiaires des effets du stress sur la sexualité. De nombreux autres peptides sont capables d'affecter le CS dans certains protocoles expérimentaux, mais leur implication physiologique n'est pas connue. Ceux dont les effets sont les plus spectaculaires sont la somatostatine, l'ocytocine, et le neu- ropeptide Y. Certaines données permettent d'espérer qu'on puisse tirer à moyen terme un bénéfice thérapeutique de manipulations de la dopamine, la noradrénaline, la sérotonine, ou des opioïdes centraux dans les dysfonctions sexuelles de l'homme. Ces progrès sont pour l'instant limités par l'importance des effets indésirables extrasexuels, eux-mêmes fonction de la spécificité insuffisante des agonistes ou antagonistes utilisés.

Mots clés : Comportement sexuel, neurotransmetteurs, neuropeptides, dopamine, noradrénaline, serotonine, béta-endorphine, opioïdes, somatostatine, ocytocine, neuropeptide $Y, C R H$, ACTH, alphaMSH, LHRH, Angiotensine II, Substance $P$.

Le rôle que jouent les hormones, avant tout la testostérone, dans le contrôle du comportement sexuel (CS) masculin est connu de longue date [12]. Celui des neurotransmetteurs (NT) aminergiques centraux, qui sont particulièrement les intermédiaires des stéroïdes sexuels pour leurs effets centraux, a été entrevu secondairement. Les 15 dernières années ont enfin permis des progrès considérables dans la cartographie des peptides et de leurs récepteurs présents dans le système nerveux central (SNC). Certains sont présents dans des régions capitales pour le contrôle du CS, et leur concentration y est régulée, comme celle des NT aminergiques, par les stéroïdes sexuels, rendant hautement probable leur propre impli- 
cation dans ce contrôle. Ce domaine a fait l'objet, ces dernières années, d'une recherche considérable, dans l'espoir d'élucider la physiopathologie des dysfonctions sexuelles (DS), et d'améliorer leur traitement en s'aidant de manipulations pharmacologiques de ce contrôle central.

Neuropeptides (NP) et autres NT interviennent à 2 niveaux dans le contrôle du CS : d'une part au niveau central, cérébral, où ils sont des intermédiaires des effets des stéroïdes et du stress, ainsi que probablement d'autres émotions et de l'affectivité. D'autre part, au niveau périphérique, pelvien et surtout intrapénien, où ils jouent un rôle fondamental d'effecteurs dans le contrôle de l'érection et de l'éjaculation. Cette mise au point n'envisagera que le niveau central.

\section{GENERALITES}

\section{Complexité du contrôle central du comportement sexuel $[21,50]$ :}

La liste des NT centraux, et particulièrement des NP, capables d'influencer le CS dans des conditions expérimentales est considérable et ne fait que croître. Tous ne sont pas pour autant forcément impliqués physiologiquement dans ce contrôle. Plus qu'un effet déterminant direct, les systèmes neuronaux qui sécrètent ces NT modulent un état d'équilibre réciproque entre les différents groupes concernés, lui-même régulièrement modifié par leurs multiples interactions. C'est par l'intermédiaire de telles variations subtiles de l'équilibre des différents systèmes neuronaux que s'exerceraient les effets des émotions, du stress [36], peut-être de l'affectivité, et en tous cas des stéroïdes sexuels sur le CS. Les stéroïdes affectent en effet profondément le métabolisme de nombreux NT. Par exemple, ils modifient à la fois la synthèse, le transport, la libération dans la synapse, la recapture et la dégradation des NT aminergiques [44]. Le même stéroïde peut exer- cer des effets opposés sur différents paramètres du métabolisme d'un même NT, ou sur des groupes neuronaux sécrétant le même NT mais situés dans des aires cérébrales différentes. De même, un même NT peut exercer des effets diamétralement opposés sur le CS selon le type de récepteur mis en jeu, sinon même selon la quantité qui en est libérée, ou la durée de sa libération.

Cette complexité explique en partie les difficultés de l'étude des effets des NT sur le CS animal et plus encore humain. Le fait que les mêmes NT et NP soient impliqués à la fois dans le SNC et dans les structures périphériques régulant plus spécifiquement érection et éjaculation augmente encore les difficultés à préciser le rôle propre des structures centrales en cas de manipulation pharmacologique. Enfin aucun NT, comme aucun type précis de récepteur, ne semble spécifique du contrôle du CS. Par exemple, les mêmes NT aminergiques centraux sont également impliqués dans les comportements d'appétit, de vigilance, et dans les différentes régulations neurovégétatives ou motrices [44]. Pour cette raison les applications thérapeutiques de leur manipulation pharmacologique sont encore très limitées, la quasi-impossibilité d'impact spécifique après administration systémique étant à l'origine d'importants effets indésirables extrasexuels.

\section{Evolution des méthodes d'explora- tion [50] :}

Les progrès des méthodes immunologiques ont permis d'affiner la cartographie des systèmes neuronaux en fonction des NT qu'ils sécrètent (immunohistochimie), et de quantifier leurs sécrétions en les dosant (radioimmunologie). Les premières études du rôle joué par ces systèmes dans le contrôle du CS ont consisté à observer les effets sexuels de manipulations pharmacologiques globales de la synthèse ou du métabolisme du NT étudié. On a également administré par 
voie générale, ou dans les ventricules cérébraux, des agonistes ou des antagonistes. Ont également été réalisées à différentes phases de l'activité sexuelle des études immunohistochimiques des zones cérébrales impliquées dans le contrôle du CS selon les résultats d'expériences stéréotaxiques de stimulation ou de destruction, ainsi que des zones dont des études autoradiographiques avaient montré la capacité d'accumuler les stéroïdes marqués administrés par voie générale. On a enfin dosé dans le liquide céphalo-rachidien les métabolites des principaux NT aux différentes phases de l'activité sexuelle.

Ces méthodes étaient cependant souvent trop grossières pour qu'on puisse interpréter sans réserve leurs résultats, comme le démontrèrent ultérieurement, par exemple, les expériences d'injection intracèrèbrale d'agonistes, capables d'induire des effets diamétralement opposés selon le site d'administration, le type de récepteur mis en jeu, la quantité administrée, ou la durée de son administration. Il fallait évoluer vers des méthodes plus fines et spécifiques, permettant d'étudier individuellement chaque groupe neuronal, chaque type de récepteur, et chaque aspect du CS. Les progrès techniques les plus importants ont comporté, outre des techniques de microinjection ou de microperfusion in vivo d'agoniste ou d'antagoniste de chaque type de récepteur dans des aires cérébrales très précises, la voltamétrie et la microdialyse [45]. Ces 2 méthodes ont permis de monitoriser chez l'animal vivant, libre dans sa cage, et susceptible d'y exercer différents types d'activité, la libération et le métabolisme des NT mono-aminergiques dans des aires cérébrales très localisées (Figure 1). Elles nécessitent l'implantation in situ, dans des zones riches en NT, de chambres de microdialyse où diffusera le milieu extracellulaire qu'on pourra aspirer et analyser, ou de micro-électrodes mesurant un voltage qui renseigne sur le potentiel oxydatif des molécules en présence, lequel est

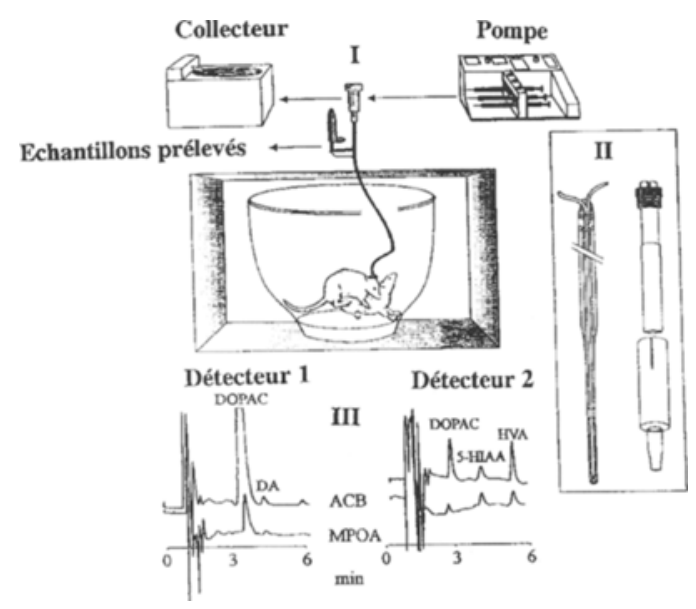

Figure 1 : Etude par microdialyse des animaux au cours d'activité sexuelle. I. Installation générale. II. Sonde de microdialyse, et canule-guide permettant le remplacement indolore de la sonde. La partie inférieure est cimentée au crâne. III. Exemples de chromatogrammes de dialysats collectés à partir de 2 aires cérébrales différentes dont l'aire préoptique médiane (MPOA) et enregistrés par un double détecteur à très haute sensibilité (d'après Mas et al [43]).

spécifique de chaque NT et permet de les identifier. Les taux des NT aminergiques peuvent ainsi être suivis en continu dans différentes zones du cerveau pendant l'acte sexuel.

Plus récemment, les progrès de la biologie moléculaire ont ouvert de nouvelles perspectives. Il s'est agi d'abord de l'identification immunocytochimique de la protéine cfos, produite très précocement après l'activation d'un gène, qui permet d'établir avec beaucoup plus de précision la cartographie des neurones activés au cours de l'activité sexuelle. On commence aussi à utiliser dans ce domaine des oligonucléotides antisens, capables de stopper la transcription d'un gène précis, par exemple celui qui contrôle la synthèse d'un NT particulier. On observe ensuite les effets de la carence ainsi induite. 


\section{Principales composantes du CS, et topographie de leur contrôle central :}

L'expérimentation animale a conduit à distinguer 2 grandes composantes [5, 24] : l'une "appetitive" ou motivationnelle, correspondant à l'excitation qui conduit le mâle à rechercher une partenaire sexuelle réceptive et a engager la copulation. Soit dans l'espèce humaine la motivation, ou appétit, ou intérêt sexuels (IS) ; d'autre part une composante "consommatoire", copulatoire, ou de performance, qui correspond à l'érection, l'intromission et l'éjaculation. Le contrôle neuro-endocrinien de ces 2 composantes est nettement différencié.

Il y a aujourd'hui un consensus sur le fait que les neurones de l'aire préoptique médiane et de l'hypothalamus antérieur (APO-HA) qui la continue jouent un rôle capital dans la régulation des aspects consommatoires $\mathrm{du}$ CS des vertébrés mâles. La situation est plus discutée en ce qui concerne les aspects motivationnels. On considère généralement que les circuits qui les contrôlent incluent principalement des circuits limbiques allant de l'amygdale ventrolatérale au striatum ventral [24]. L'amygdale ventrolatérale est impliquée dans le traitement des informations provenant du cortex et du système olfactif, ainsi que dans les réactions au stress comme la peur [36]. Des données récentes suggèrent cependant que l'APO-HA intervient également dans certains aspects motivationnels comme le choix d'une partenaire de l'autre sexe et sa poursuite [5].

\section{NEUROTRANSMETTEURS MONO-AMINERGIQUES}

Il s'agit des catécholamines type dopamine (DA), noradrénaline (NA) et adrénaline, et des indolamines type sérotonine (ST).

\section{Dopamine (DA) :}

\section{a) Expérimentation animale $[24,36]$ :}

La DA et ses agonistes, particulièrement l'apomorphine dont l'effet s'exerce à la fois sur récepteurs dopaminergiques D1 et D2, stimulent globalement les CS du rat [24] et du chien [41]. Son effet est cependant biphasique. Il devient inhibiteur à forte dose [36], peut-être du fait d'une désensibilisation des récepteurs. Les études in situ réalisées chez le rat (perfusions, microdialyse, voltamétrie) suggèrent des sites séparés pour le contrôle de la composante appétitive (nucleus accumbeus du striatum ventral), et celui de la composante consommatoire (APO-HA comme pour les opiacés. Castration et supplémentation en testostérone modifient les taux de DA, ainsi que les récepteurs dopaminergiques D2L dans l'APO-HA du rat mâle [30].

Chez le singe rhésus, la DA facilite également les érections et la masturbation, plus particulièrement en présence d'une femelle [51]. Cet effet dépend alors de la stimulation des récepteurs D2, à l'exclusion des récepteurs D1. Par contre, la stimulation des récepteurs tant D1 que D2 tend à inhiber l'éjaculation [51], qui est au contraire stimulée par la DA chez le chien [41].

\section{b) Données chez l'homme [62] :}

La DA pourrait également exercer des effets stimulants dans l'espèce humaine.De nombreuses publications anecdotiques ont rapporté la survenue d'une stimulation du CS (IS et érection) chez les parkinsoniens traités par agonistes dopaminergiques ( $\mathrm{L}$ dopa, Pergolide, Amantadine) mais aucune donnée objective n'a confirmé cet effet [59]. L'étude ouverte de Benkert [7] n'a pas trouvé d'avantage thérapeutique à la L-dopa dans les dysfonctions érectiles (DE) et manques d'intérêt sexuel (MIS) psychogènes. Un antidépresseur non tricyclique, le buproprion, qui agit indirectement comme un agoniste dopaminergique en inhibant la recapture de la DA, aurait augmenté l'IS et le degré de satisfaction sexuelle dans différents types de DS [17]. Cet effet n'a pas été retrouvé dans une autre étude portant sur des sujets avec MIS [Klein et al in 59]. Enfin un psychotrope 
antidopaminergique, le benpéridol, inhibe clairement l'IS chez l'homme [65]. Bien qu'il ne modifie pas le taux des androgènes, son effet est identique à celui des antiandrogènes.

Les données les plus significatives proviennent cependant de l'injection sous cutanée d'apomorphine, agoniste spécifique D1 + $\mathrm{D} 2$, qui induit en 10 à $20 \mathrm{mn}$ une érection chez deux tiers des hommes normaux et des impuissants idiopathiques, sans modifier l'IS [43, 62]. Plusieurs études ont montré que cet effet est significativement supérieur à celui d'un placebo, et semble s'exercer au niveau central puisqu'il est inhibé par les antagonistes de la DA à impact central, mais pas par ceux qui ont un impact périphérique comme la dompéridone [62]. Une étude récente suggère cependant que chez le rat, le site d'action de l'apomorphine serait en fait le système nerveux périphérique, plus particulièrement le système autonome [54].

Les injections d'apomorphine induisent des nausées, une hypotension, ou des vertiges chez plus de $50 \%$ des hommes, ce qui empêche leur utilisation thérapeutique. Récemment Heaton est cependant parvenu à réduire la fréquence de ces effets secondaires jusqu'à un niveau très faible, tout en gardant une réponse sexuelle valable, chez 60 à $80 \%$ des sujets grâce à une nouvelle formulation permettant une absorption sublinguale [35]. Lal a rapporté que la majorité des répondeurs à l'apomorphine étaient améliorés par la bromocriptine, un autre agoniste dopaminergique, mieux toléré et actif per os [in 62]. Cependant son étude ne comportait pas de groupe témoin, et ses résultats n'ont pas été confirmés par la pratique clinique. Particulièrement la bromocriptine ne s'est pas montrée supérieure à un placebo chez les impuissants idiopathiques non sélectionnés [in 14].

La voie dopaminergique pourrait donc ouvrir des perspectives thérapeutiques intéressantes dans le traitement des DS.
Un agoniste D2 spécifique, la quinelorane, a connu un début de développement dans cette indication [29]. Il a été abandonné secondairement, probablement du fait d'un taux excessif d'effets indésirables lié à la difficulté d'agir spécifiquement sur les centres du CS.

\section{Noradrénaline :}

\section{a) Expérimentation animale :}

Chez les rongeurs, l'administration d'agonistes (clonidine) ou d'antagonistes (yohimbine, idazoxan) des récepteurs alpha 2 adrénergiques centraux induit des effets sexuels marqués en faveur de l'existence d'un tonus adrénergique central inhibant en permanence à la fois motivation et performance sexuelles par l'intermédiaires des récepteurs alpha 2 , qu'on trouve particulièrement localisés dans l'APO [14, 16, 50]. Ceci pourrait expliquer les effets sexuels iatrogènes de la clonidine, utilisée chez l'homme pour traiter l'hypertension artérielle (Catapressan $®$ ) [13]. Il faut savoir que les récepteurs alpha 2 sont généralement impliqués dans l'inhibition présynaptique de la libération de NA [37], et que c'est donc une augmentation de la libération de NA (et de l'activité des neurones noradrénergiques) qui est responsable de l'effet stimulant sexuel observé après administration d'alpha 2-antagonistes. Le tonus alpha 2-adrénergique pourrait être modulé par l'environnement stéroïdien. Ce contrôle noradrénergique est probablement intriqué avec celui qu'exercent différents neuropeptides colocalisés dans les extrémités nerveuses contenant de la NA (Neuropeptide $\mathrm{Y}$, Somatostatine, Angiotensine II) [16].

La yohimbine stimule non seulement le CS des rats normaux, mais aussi celui d'une souche de rats constitutionnellement inactifs sexuellement, et celui des rats castrés chez qui elle potentialise l'effet de faibles doses de testostérone (test.) $[14,16,26]$. Des mécanismes adrénergiques pourraient donc intervenir en aval de la test. On sait 
par ailleurs qu'au niveau même du pénis, la NA inhibe l'érection en contractant le muscle lisse caverneux après activation, cette fois, des récepteurs alpha 1 . Ceci réduit l'entrée du sang dans les aréoles caverneuses, et c'est un tonus noradrénergique permanent qui est responsable du maintien de la flaccidité par l'intermédiaire de ce mécanisme périphérique.

\section{b) Données dans l'espèce humaine :}

La NA circulante augmente tant chez l'homme que chez la femme en cas d'excitation sexuelle [67]. Ce phénomène pourrait faciliter l'éjaculation puisque les agonistes alpha-adrénergiques la stimulent en cas de dysfonction éjaculatoire type éjaculation rétrograde, tandis que les antagonistes alpha 1 allongent le délai éjaculatoire [57]. On met souvent en cause un excès de libération de NA lié au stress, et particulièrement à la peur de l'échec, à l'origine des $\mathrm{DE}$ psychogènes. L'excès de NA s'opposerait à la relaxation du muscle lisse caverneux requise pour l'érection par un impact principalement périphérique. On a trouvé une augmentation du taux de la NA dans le sang pénien des impuissants psychogènes [40]. On peut imaginer qu'un mécanisme voisin est impliqué dans le déterminisme de l'éjaculation prématurée.

Différentes études ont testé l'intérêt thérapeutique d'une manipulation des systèmes noradrénergiques centraux. Particulièrement plusieurs auteurs ont cherché récemment à confirmer les effets bénéfiques présumés de la Yohimbine, un antagoniste alpha 2 d'origine végétale utilisé depuis longtemps de façon empirique, en la soumettant à la méthodologie des essais en double insu (DI) contre placebo $[46,55,58$, $61,64]$. La Yohimbine fut utilisée à la dose de 12 à $42 \mathrm{mg} / \mathrm{j}$. Bien que chaque étude souffre de quelques insuffisances méthodologiques, l'ensemble est en faveur d'un effet positif réel, quoique discret, sur les érections coïtales et nocturnes [61], ainsi que peut-être dans l'étude de Sonda [61], sur
l'IS et l'orgasme. La supériorité statistique sur le placebo n'est que limite, au prix d'effets secondaires remarquablement modestes, ce qui est inhabituel en cas de manipulation des NT cérébraux. La même tendance à la supériorité sur le placebo a été objectivée avec un autre antagoniste alpha 2, le fluparoxan, concernant cette fois non seulement les érections mais également l'IS [57].

Bancroft [4] a montré qu'un troisième antagoniste alpha 2, la delequamine, augmentait les érections nocturnes des hommes jeunes. On sait que celles-ci surviennent au cours du sommeil paradoxal, qui se caractérise par une extinction de l'activité des neurones noradrénergiques centraux du locus coeruleus, et par une réduction de l'activité sympathique périphérique propre à faciliter les érections [49]. L'effet sur les érections nocturnes était moins marqué chez les sujets avec $\mathrm{DE}$, et chez les sujets âgés, qu'ils soient normaux ou aient une DE. Bancroft a interprété ces données comme l'indice d'une augmentation du tonus alpha 2-adrénergique central inhibiteur en cas de $\mathrm{DE}$, ainsi que de façon générale avec l'âge. Les développements thérapeutiques du fluparoxan et la delequamine ont été abandonnés faute d'efficacité clinique suffisante. La manipulation des systèmes noradrénergiques centraux, qui paraissait particulièrement intéressante vu la modicité de ses effets indésirables, s'est donc avérée plutôt décevante jusqu'à présent.

\section{Sérotonine (ST) :}

\section{a) Expérimentation animale :}

La ST a longtemps été considérée essentiellement inhibitrice de tous les aspects du CS. En effet chez le rat, l'augmentation de la transmission sérotoninergique centrale augmente la proportion des animaux non copulants, ainsi que la latence éjaculatoire. L'inhibition de cette transmission, ou la destruction des voies sérotoninergiques, a 
l'effet inverse. La stimulation sexuelle qui en résulte concerne les animaux constitutionnellement inactifs sexuellement, et les animaux castrés chez qui les substances antisérotonine potentialisent des doses minimales d'androgènes [14, 44]. La disponibilité récente d'agonistes sérotoninergiques spécifiques des différents récepteurs a cependant permis de démontrer que l'effet de la ST pouvait être diamétralement opposé selon le type de récepteur et le paramètre sexuel concernés, avec probablement une relation réciproque entre la stimulation des récepteurs 5-HT2C et 5-HT1A [51].

Ainsi chez le rat [34, 50], l'administration de 8-OH-DPAT, agoniste spécifique des récepteurs 5HT1A, augmente la fréquence de l'activité sexuelle et des éjaculations, et diminue significativement la latence éjaculatoire, induisant chez la moitié des animaux une véritable éjaculation "prématurée". Celle-ci survient après seulement 1 ou 2 intromissions contre normalement 10 à 12 , et même "ante portas", c'est-à-dire extravaginale, chez 25 à $33 \%$ des animaux testés avec les doses les plus élevées. Ce comportement est tout à fait inhabituel chez le rat. Cet effet est dépendant des androgènes. Chez le singe, le même agoniste 5-HT1A réduit également le délai éjaculatoire, mais tend à inhiber les érections survenant en présence des femelles [51]. A l'inverse, un agoniste spécifique des récepteurs 5-HT2C, le m-CPP, semble faciliter les érections et interférer avec l'éjaculation.

\section{b) Données chez l'homme :}

Dans une étude ancienne, Benkert aurait pu améliorer des sujets impuissants en associant un anti-ST à des androgènes [6]. Son étude ne peut pas cependant être interprétée sans réserve du fait de faiblesses méthodologiques. La variabilité des effets des agonistes selon le type de récepteur concerné pourrait expliquer le caractère apparemment contradictoire des différents effets indésirables sexuels qu'on a rapportés avec une fréquence élevée (10 à $30 \%$ des sujets traités) au cours de traitements de dépressions, de syndromes obsessifscompulsifs, d'attaques de paniques ou de boulimies nerveuses par des inhibiteurs de la recapture de la ST $([20,59])$ : il s'est agi le plus souvent d'inhibitions sexuelles caractérisées par une éjaculation ou un orgasme retardés, jusqu'à l'anéjaculation sans orgasme, un MIS ou une anesthésie génitale. Mais on a aussi observé la correction inopinée de $\mathrm{DE}$, des érections prolongées, ainsi qu'un engorgement clitoridien et des orgasmes multiples en l'absence de toute stimulation sexuelle.

L'allongement du délai éjaculatoire induit par la chlorimipramine (Anafranil@), antidépresseur tricyclique à impact sérotoninergique marqué (10 à $30 \mathrm{mg} / \mathrm{j}[3,34])$ et par les nouveaux inhibiteurs de la recapture de la ST (Fluoxetine, Prozac ${ }^{\circledR} 20 \mathrm{mg} / \mathrm{j}$ $[52,63])$ est aujourd'hui couramment utilisé pour traiter l'éjaculation prématurée. On les administre soit de façon continue, soit en prise unique le matin du rapport, la chlorimipramine, qui a simultanément un effet adrénolytique périphérique, ayant fait la preuve de son efficacité selon les 2 schémas dans des études en double insu contre placebo. Le bénéfice ne semble concerner que l'éjaculation prématurée pure, sans $\mathrm{DE}$ associée [34].

Un autre inhibiteur de la recapture de la ST, la trazodone (Pragmarel®) a également été proposé dans le traitement des $\mathrm{DE}$. De nombreux priapismes ont été observés au cours de traitements de déprimés sans DE par cet antidépresseur, qui influence également les systèmes adrénergique et dopaminergique [60].Un effet stimulant de l'IS indépendant de l'effet antidépresseur a également été rapportée dans plusieurs études ouvertes [59].Deux études en DI contre placebo menées chez des hommes normaux ont également conclu à une augmentation de la durée des érections nocturnes peut-être attribuable à l'effet alpha-bloqueur simultané de la trazodone [59]. En cas de DE, à 
la dose de 50 à $150 \mathrm{mg} / \mathrm{jour}$, la trazodone s'est montrée plus active qu'un placebo dans 2 études [2, 42] mais non dans une troisième [19]. Son utilisation est cependant limitée par son impact sur la vigilance, qui rend préférable une prise unique, vespérale.

La manipulation des systèmes sérotoninergiques a donc probablement un avenir thérapeutique, peut-être plus spécifiquement dans le domaine du traitement de l'EP.

\section{Conclusions :}

Les données précédentes suggèrent que les systèmes mono-aminergiques centraux gardent dans l'espèce humaine un rôle important dans la modulation du CS. Ils sont probablement des intermédiaires d'une partie des effets des émotions et des stéroïdes sexuels sur la sexualité. Mais du fait que l'implication de ces systèmes déborde largement la sphère sexuelle, les applications thérapeutiques de leur manipulation pharmacologique restent encore aujourd'hui limitées par d'importants effets indésirables non sexuels.

\section{NEUROPEPTIDES CEREBRAUX}

La liste des neuropeptides susceptibles de modifier le CS est déjà très longue et ne cesse de croître. Je ne citerai ici que les principaux. Comme nous le verrons, certains ont des effets très importants.

\section{Corticotropin Releasing Hormone (CRH, [21]) :}

Cette hormone hypothalamique active la réponse hypophyso-surrénalienne au stress, et inhibe puissamment la fonction reproductrice. D'une certaine façon ses effets s'opposent à ceux de la Luteotropin Hormone Releasing Hormone (LHRH). Ils s'exercent en bonne part via les sécrétions d'AC$\mathrm{TH}$ et d'opioïdes, particulièrement de bétaendorphine (voir plus loin). Le CRH semble également capable d'inhiber directement la sécrétion de LH. Il semble de plus en plus évident que ce NP est responsable non seulement de la réponse endocrinienne, mais également des réponses comportementales et neurovégétatives au stress.

Sirinathsinghji (in [21]) a montré que l'injection intraventriculaire de $\mathrm{CRH}$ inhibait le comportement copulatoire du rat mâle. Cet effet sexuel semble cependant médié par la stimulation de la sécrétion de bétaendorphine, car il est prévenu par l'administration simultanée d'un antagoniste, la Naltrexone. Il éclaire sur ce qui constitue probablement l'un des principaux mécanismes des effets du stress sur le CS (d'autres pouvant impliquer les catécholamines et la prolactine).

2. Substances opioïdes $[5,14,21,24,36$, 47] :

Il s'agit des opiacés endogènes, doués d'effets de type morphinique et dérivés, comme l'ACTH et la MSH, d'une grosse molécule, la pro-opio-mélano-cortine (Figure 2), sous l'effet du $\mathrm{CRH}$, lui-même particulièrement libéré en réponse au stress. Les principaux opioïdes isolés sont la béta-endorphine, l'enképhaline, la leu-enképhaline, la dynorphine. On en trouve dans de nombreux sites de l'organisme, y compris dans les testicules. Mais leur synthèse est principalement cérébrale. Ils ont une double fonction de NT et d'hormones, puisque libérés dans la circulation générale et susceptible d'effets à distance. Ils participent à de nom-

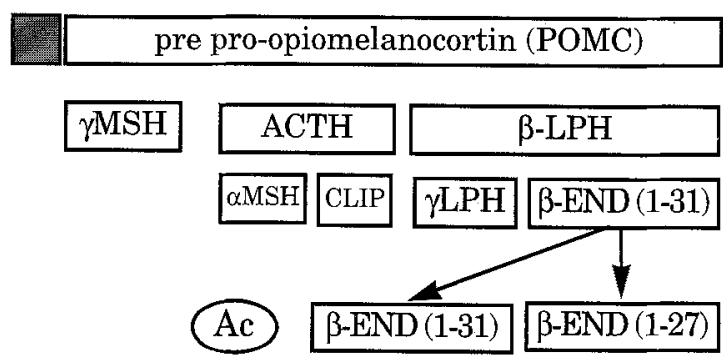

Figure 2 : Synthèse des substances opiö̈des dans le cerveau. B-END : béta-endorphine. $B$-LPH : béta-lipotrophine. 
breuses fonctions de l'organisme, incluant le contrôle de la fonction gonadique qu'ils tendent à inhiber en réduisant la sécrétion de $L H$ et stimulant celle de prolactine (PRL). De nombreux arguments suggèrent leur intervention dans le contrôle du CS.

\section{a) Effets des opiacés et des opioüdes :}

Chez l'animal, leur administration par voie générale réduit la motivation et surtout la performance sexuelles (copulation). Cet effet est spécifique et ne concerne pas d'autre fonction que sexuelle. La perfusion de quantités minimes de béta-endorphine dans l'APO du rat induit des effets identiques à ceux de la destruction de cette aire, c'est-à-dire une suppression de la copulation en dépit d'une motivation sexuelle normale (recherche et explorations actives des femelles en estrus). L'effet inhibiteur de la perfusion disparaît si elle est instaurée après une première intromission, sauf si on présente au rat une nouvelle femelle à laquelle il n'est pas habitué. Ceci suggère que plus que le comportement copulatoire lui-même, c'est le processus par lequel l'information sensorielle récoltée pendant la phase «appétitive» est traitée et active le comportement copulatoire qui est inhibé par la béta-endorphine. Cette déconnection pourrait être l'un des mécanismes fondamentaux de la diminution du comportement copulatoire observé en réponse au stress chez le rat et peut-être aussi chez l'homme, cette situation expérimentale chez le rongeur ayant pu être considérée comme une sorte de modèle expérimental de l'impuissance psychogène [25].

A l'inverse, une perfusion de béta-endorphine dans l'amygdale du rat inhibe la phase appetitive (recherche et exploration de la femelle) et retarde l'initiation de la copulation sans cependant l'inhiber. Cet opioïde peut donc inhiber les 2 principales composantes du CS en fonction de son site d'intervention, tout comme il peut inhiber la fonction gonadique elle-même.
Les données dont on dispose dans l'espèce humaine sont plus restreintes. L'utilisation chronique d'opiacés diminue l'IS et les rêves érotiques, perturbe les érections, retarde l'éjaculation, et diminue plaisir sexuel et pollutions nocturnes.

\section{b. Effets des antagonistes des opiacés chez l'animal et chez l'homme normal :}

Dans la majorité des protocoles expérimentaux, Naloxone et Naltrexone induisent ou facilitent le CS. Ces antagonistes suppriment de façon spectaculaire les effets inhibiteurs des opioïdes. Perfusée dans l'APO$\mathrm{HA}$, la naloxone stimule puissamment le comportement copulatoire. Chez le singe et chez l'homme, on a également observé la survenue d'érections dans des situations non sexuelles après administration d'antagonistes, encore qu'Abbott et coll [25] aient au contraire observé une inhibition sexuelle totale et prolongée chez le singe.

L'effet le plus spectaculaire des antagonistes est d'induire un CS normal chez les rats constitutionnellement inactifs sexuellement, qui constituent un modèle animal du MIS humain. Cet effet a particulièrement été retrouvé dans une souche de rats présentant également constitutionnellement obésité et taux élevé de béta-endorphine, mais pas dans 2 études consacrées à des singes constitutionnellement inactifs sexuellement $[1,31]$.

\section{c. Effets des antagonistes chez l'impuis- sant :}

Deux études en double insu contre placebo $[11,27]$, et 2 autres ouvertes [28, 32], ont administré oralement de la Naltrexone à des impuissants non organiques. Toutes ont rapporté une augmentation des érections matinales, significativement plus importante que sous placebo dans les 2 études concernées. Goldstein a aussi observé une amélioration des érections nocturnes mesurées par plethysmographie [32]. Aucun effet sur la libido et aucune modification hormonale n'ont été observées. Trois des 4 études ont rapporté 
une nette amélioratior des érections coïtales, dans l'une supérieure aux effets du placebo [27]. Mais dans l'étude de Frajese [28], cet effet bénéfique, obtenu seulement avec les doses les plus élevées, fut transitoire, et diminua à partir de la 4ème semaine. De plus, la seconde étude contre placebo ne put confirmer l'effet bénéfique [11].

\section{d) Opioüdes et éjaculation :}

In vitro, les opioïdes inhibent les contractions du canal déférent et des vésicules séminales. Ils pourraient donc jouer un rôle dans la régulation de la fertilité masculine en modulant le volume de l'éjaculat et le nombre de spermatozoïdes éjaculés. Certaines données suggèrent aussi qu'ils pourraient moduler la latence éjaculatoire, réduite chez les rongeurs par les antagonistes des opioïdes.

\section{e) Mécanismes des impacts sexuels des opioïdes :}

- Impact direct : il est suggéré par la découverte chez l'animal de récepteurs spécifiques en grand nombre dans les aires cérébrales contrôlant le CS, et démontré par les résultats des injections in situ déjà décrites.

- Médiation endocrinienne : elle est plausible puisque les opioïdes affectent les 2 principales hormones à impact sexuel, la test. qu'ils diminuent en inhibant la sécrétion pulsatile de LH, et la PRL, dont ils stimulent la sécrétion.

- Médiation par les autres NT cérébraux : elle est également plausible puisque les opioïdes inhibent l'activité des systèmes dopaminergiques qui facilitent le CS, et augmentent celle des systèmes sérotoninergiques qui peuvent l'inhiber.

\section{f) Conclusion :}

Les opioïdes sont impliqués à la fois dans le contrôle du CS et dans celui de la fertilité, qu'ils inhibent chez l'animal comme dans l'espèce humaine. Expérimentalement, ils sont susceptibles d'inhiber les 2 principales composantes du CS, motivation et performance. Cependant ces effets pourraient éventuellement être dissociés puisqu'ils mettent en jeu 2 aires cérébrales différentes. Les résultats obtenus par la Naltrexone dans les DE psychogènes semblent limités par les effets indésirables qui apparaissent à forte dose, ou après utilisation prolongée, et par une labilité spontanée des effets dans le temps. Celle-ci s'explique probablement par la reconstitution de nouveaux équilibres entre les différents systèmes de NT. Ils ouvrent cependant des perspectives intéressantes.

L'hypothèse selon laquelle les opioïdes constitueraient des intermédiaires importants des effets du stress et de l'anxiété sur l'IS et/ou la fonction érectile est très séduisante. Stress et anxiété augmentent effectivement leur libération [53]. Chez la femme, le rôle qu'ils jouent dans l'articulation psychosomatique des aménorrhées psychogènes est démontré.

\section{Adreno-Corticotropin-Hormone (ACTH), Alpha Melano-Stimulating- Hormone (Alpha MSH) :}

Ces deux peptides sont également libérés à partir de la pro-opio-melano-cortine sous l'effet du CRH, particulièrement en réponse au stress. Ils favorisent la composante appétitive du CS, ce qui n'est peut être qu'un aspect non spécifique de leur effet général motivant $[5,10,21]$. Ils stimulent également la composante consommatoire, diminuant chez le rat la latence éjaculatoire $[9,24]$ et induisant l'érection lorsqu'on les injecte dans les ventricules. Cet effet est plus net pour l'alpha-MSH [9, 21]. Il pourrait exister un antagonisme entre ces peptides et les opioïdes, en dépit de leur origine commune. Leur accumulation en grande quantité dans l'hypothalamus des animaux chroniquement intoxiqués par les opiacés pourrait être responsable des érections et éjaculations survenant lors du sevrage [9]. 


\section{Luteotropin Hormone Releasing Hormone (LHRH) :}

Clé de voûte du contrôle de la fonction gonadique, ce neuropeptide présent dans les terminaisons nerveuses de l'APO-HA exerce également un effet stimulant direct sur le CS des rongeurs, y compris après injection intraventriculaire. Cet effet persiste chez l'animal castré ou hypophysectomisé à condition qu'il soit supplémenté en stéroïdes $[10,21]$. La LHRH stimule aussi la proceptivité chez le singe femelle, même castré (s'il est supplémenté en stéroïdes) [39]. Dans l'espèce humaine, des études en double insu réalisées chez l'homme normal [23] ou impuissant [8] ont confirmé un effet stimulant discret sur l'IS et les érections spontanées, trop faible pour un bénéfice thérapeutique significatif [18, 22]. Au total la LHRH semble garder un discret effet favorisant sexuel dans l'espèce humaine, sans intérêt pratique thérapeutique. On s'est posé la question de la responsabilité de la diminution de son taux dans le cerveau des rats ,âgés dans leur déclin sexuel [in 21].

\section{Somatostatine [16] :}

Ce peptide qui inhibe physiologiquement la sécrétion de l'hormone de croissance a été identifié dans différentes aires cérébrales impliquées dans le contrôle du CS. Les mécanismes somatostatinergiques pourraient être influencés par les stéroïdes gonadiques et interagir avec les fonctions noradrénergiques car NA, somatostatine et Neuropeptide Y sont souvent colocalisés dans les mêmes extrémités nerveuses.

Chez le rat, des modifications des taux intracérébraux de ce peptide sont associées à des modifications du comportement copulatoire, aboli lorsqu'il augmente, et renforcé lorsqu'il diminue. Ces effets semblent particulièrement s'exercer au niveau de l'APO comme le montrent les expériences de micro-injection in situ. On ne dispose jusqu'à présent d'aucune donnée dans l'espèce humaine.

\section{Ocytocine $[14,15,21,48,50]$ :}

Ce NP est sécrété par l'hypophyse postérieure, et tant la test. que les ostrogènes stimulent la formation de ses récepteurs. Ses principaux effets physiologiques sont de stimuler les contractions utérines lors de l'accouchement, ainsi que l'éjection du lait lors de l'allaitement par l'intermédiaire d'une contraction des muscles lisses des canaux galactophores. Chez le rat et le mouton, l'ocytocine est également impliquée dans le comportement maternel.

Dés 1976, Fox, puis Newton [in 15] suggéraient un rôle de ce NP dans l'orgasme féminin. Celui-ci survient occasionnellement au cours de l'accouchement et de l'allaitement, et est sous-tendu par des contractions de l'utérus et du périnée. La mise au point de dosages radio-immunologiques fiables a également permis de suspecter un rôle physiologique chez l'homme. L'ocytocine circulante augmente graduellement au cours de l'excitation sexuelle chez l'homme comme chez la femme, pour culminer au moment de l'éjaculation et de l'orgasme $[15,48,66]$. Son taux atteint alors des valeurs de l'ordre de celles de l'allaitement. L'ocytocine pourrait avoir dans ces conditions un rôle facilitateur du transport des gamètes, puisqu'elle augmente la contractilité des épididymes, déférents, et canaux éjaculateurs.

En objectivant un triplement des taux d'ocytocine dans le liquide céphalo-rachidien du rat, Hugues [38] a pu montrer que ce NP pouvait également être libéré dans le SNC au cours de l'activité sexuelle. Toujours chez le rat, l'administration périphérique ou intraventriculaire d'ocytocine a un effet facilitateur (quoique parfois inhibiteur à forte dose) sur la performance sexuelle, tandis que l'administration d'un antagoniste de ce NP a un effet inhibiteur spectaculaire de l'aspect consommatoire du CS $[21,50]$.

L'ocytocine semble donc avoir chez l'animal d'un effet facilitateur de la performance 
sexuelle (érection, éjaculation), sans qu'on sache si cet effet a des implications physiologiques. Dans l'espèce humaine, Riley [56] a testé sans succès ce NP chez des éjaculateurs retardés et des anéjaculateurs sans orgasme.

\section{Arginine Vasopressine (AVP) ou hor- mone antidiurétique :}

Chez le rat, l'AVP favorise le maintien d'une activité sexuelle après castration. On interprète ce phénomène comme une conséquence non spécifique de sa capacité à renforcer la mémorisation des comportements appris [10]. L'AVP pourrait cependant également être impliquée dans les mécanismes d'adaptation au stress chronique. Ainsi le contenu en AVP des neurones à CRH du rat augmente en cas d'immobilisation forcée prolongée (in [36]). On ne dispose pas de données dans l'espèce humaine.

\section{Angiotensine II [16] :}

Le rôle physiologique principal de ce peptide est la régulation de l'équilibre hydroelectrolytique et de la pression artérielle. Il semble cependant de plus en plus clair qu'il joue aussi un rôle important dans le contrôle des fonctions reproductrices. L'angiotensine II semble inhiber le comportement copulatoire indépendamment de sa capacité à stimuler le besoin de boire, comme l'ont montré les expériences d'injection intraventriculaire, et la facilitation du CS qui résulte du blocage de ses récepteurs. Les implications physiologiques ne sont pas connues, mais toujours chez le rat, l'administration chronique d'un inhibiteur de l'enzyme de conversion ou d'un bloqueur des récepteurs de l'angiotensine n'a pas d'effet sur le CS.

\section{Melatonine [33] :}

Principale hormone sécrétée par l'épiphyse ou glande pinéale, la mélatonine est responsable des variations saisonnières du comportement reproducteur d'un grand nombre d'espèces animales. Par l'intermé- diaire de ses effets sur les systèmes opioïdes et catécholaminergiques, elle inhibe la sécrétion de la LHRH, donc la fonction gonadique, et le CS. Son rôle physiologique dans l'espèce humaine est mal connu. L'hypothèse d'une augmentation de la mélatonine dans l'impuissance psychogène, ou idiopathique, a été testée. Si l'on a trouvé une augmentation de la réponse de cette hormone à l'administration de LHRH, ses taux circulants, comme son rythme nycthemeral, ont été trouvés normaux.

\section{Neuropeptide $Y[16,21]$ :}

Ce peptide très abondant dans le cerveau du rat est particulièrement trouvé dans l'APO-HA. Il module la fonction catécholaminergique, et est d'ailleurs souvent colocalisé avec la NA dans les terminaisons nerveuses. Il influence également la sécrétion des gonadotrophines, et exerce, au niveau périphérique une activité vaso-constrictive puissante peut être impliquée dans la détumescence pénienne.

Chez le rat, son administration par voie générale stimule puissamment le comportement alimentaire, et bloque simultanément, de façon spectaculaire, le comportement copulatoire après un début de monte, comme s'il ne permettait pas le maintien d'un niveau suffisant d'excitation pour terminer l'acte sexuel. Cependant son administration locale aboutit à des effets différents selon le site d'injection (réduction plus modeste de l'activité copulatoire après injection dans l'APO-HA ; au contraire facilitation de l'éjaculation après injection dans le noyau paraventriculaire). Clark a mis en cause la diminution avec l'âge de l'activité des neurones à Neuropeptide Y du noyau paraventriculaire dans les difficultés à éjaculer du rat ,âgé [16]. On ne dispose d'aucune donnée dans l'espèce humaine. Les effets de ce neuropeptide chez le rat rappellent cependant certaines inhibitions du CS associées à une hypertonie du comportement alimentaire observées en clinique. 


\section{Substance $P[21]$ :}

Ce neuropeptide est trouvé en abondance dans un circuit impliqué dans le contrôle du $\mathrm{CS}$, et comportant de nombreuses cellules capables d'accumuler les stéroïdes. Il inclut, outre l'APO-HA, l'amygdale médiane et le lit de la strie terminale. Son abondance y est fonction du taux circulant des stéroïdes, et diminue après castration. L'injection de substance $\mathrm{P}$ dans l'APO-HA a un effet facilitateur sexuel qui dépend $\mathrm{du}$ niveau circulant des androgènes. Cet effet facilitateur dépend peut-être de la libération de LHRH que pourrait induire la substance $\mathrm{P}$ au niveau de l'éminence médiane.

\section{CONCLUSIONS}

Des progrès substantiels ont été accomplis ces dernières années dans l'analyse des effets sexuels des manipulations des NT mono-aminergiques et peptidergiques chez les animaux, et à un moindre degré chez l'homme. Les données dont on dispose ressemblent cependant à un patchwork, et des lacunes énormes persistent dans notre compréhension de la façon dont ces différents rouages s'assemblent pour coder le CS, ou plus simplement dans la compréhension du rôle fonctionnel joué par chaque NT dans l'équilibre d'ensemble, et dans le repérage des contextes environnementaux ou comportementaux particuliers qui président à sa mise en jeu. Quelques données pharmacologiques recueillies dans l'espèce humaine, tout particulièrement suite à la manipulation des systèmes mono-aminergiques et opioïdes, sont cependant encourageantes. Elles suggèrent qu'à défaut de comprendre parfaitement ce que nous faisons quand nous réalisons ces manipulations, le temps n'est peut-être plus très éloigné où nous pourrons en tirer un bénéfice thérapeutique, que la manipulation ait concerné un système précis ou un ensemble de systèmes.

Remerciements : A Mlle Nathalie BARLET pour la préparation du manuscrit.

\section{REFERENCES}

1. ABBOTT D.H., HOLMAN S.D., BERMAN M., NEFF D.A., GOY R.W. : Effects of opiate antagonists on hormones and behavior of male and female rhesus monkeys. Arch. Sex. Behav., 1984, 13 : 126.

2. ALBO M., SWERS W.D. : Oral trazodone as initial therapy for the management of impotence ; J. Urol., 1993, 149 : 344A.

3. ASSALIAN P. : Clomipramine in the treatment of premature ejaculation ; J. Sex. Res., 1988, 24 : 213-215.

4. BANCROFT J. : Effects of alpha 2 antagonists on male erectile response ; In : The pharmacology of sexual function and dysfunction. J. Bancroft ed. Excerpta medica, Amsterdam, 1995, 6 : 215-221.

5. BAUM M.J. : Reassessing the role of medical preoptic area/anterior hypothalamic neurons in appetitive aspects of masculine sexual behavior ; In : The pharmacology of sexual function and dysfunction. J. Bancroft ed. Excerpta medica, Amsterdam, 1995, $6: 133-139$.

6. BENKERT O. : Pharmacological experiments to stimulate human sexual behavior ; In : Psychopharmacology of sexual disorders and drug abuse. T.A. Ban, North Holland, Amsterdam, 1973, 489-495.

7. BENKERT O., CROMBACH G., KOCKOTT G. : Effect of L-dopa on sexually impotent patients ; Psychopharmacologia, 1972, 23 : 91-95.

8. BENKERT O., JORDAN R., DHALEN H.G., SCHNEIDER H.P.G., GAMMEL G. : Sexual impotence : a double-blind study of LHRH-nasal spray versus placebo. In : Neuropsychobiology. 1975, 1 : 203-210.

9. BERTOLINI A., FRATTA W., GESSA G.L., MONTALDO S., SERRA G. : Penile erection during morphine withdrawal : possible role of ACTHMSH peptides. In : Central and peripheral endorphins. Basic and clinical aspects, Raven Press New York, 1979, 365-388.

10. BOHUS B. : The influence of pituitary neuropeptides on sexual behavior ; In : Hormones et sexualité. H.P. Klotz ed. Expansion Scientifique Française, Paris, 1977, 235-246.

11. BRENNEMANN W., STITZ B., VAN AHLEN H., BRENSING A., KLINGMÜLLER D. : Treatment of idiopathic erectile dysfunction in men with the opiate antagonist naltrexone-A double-blind study ; J Andrology, 1993, 14 : 407-410.

12. BUVAT J. : Hormones et comportement sexuel de l'homme : données physiologiques et physiopathologiques. Contr. Fertil. Sex. 1996, sous presse. 
13. BUVAT J., BUVAT-HERBAUT M., LEMAIRE A., KORNOBIS C. : Perturbations sexuelles masculines d'origine médicamenteuse. In : Perturbations iatrogènes de la sexualité ; J. Buvat et J. Hermabessière eds, Progrès en Andrologie I, Doin, Paris, 1987, 48-60.

14. BUVAT J., BUVAT-HERBAUT M., LEMAIRE A., MARCOLIN G. : Hormones, neurotransmetteurs centraux et comportement sexuel de l'homme. In : Andrologie III, Arvis G. ed., Maloine, Paris, 1991 : 1161-1189.

15. CARMICHAEL M.S., HUMBERT R., DIXEN J., PALMISANO G., GREENLEAF W., DAVIDSON J.M. : Plasma oxytocin increases in the human sexual response. J. Clin Endocrinol. Metab., 1987, $64: 27-31$.

16. CLARK J.T. : Sexual arousal and performance are modulated by adrenergic-neuropeptide-steroid interactions ; In : The pharmacology of sexual function and dysfunction. J. Bancroft ed. Excerpta medica, Amsterdam, 1995, $6: 55-68$.

17. CRENSHAW T.L., GOLDBERG J.P., STERN W.C. : Pharmacologic modification of psychosexual dysfunction ; J. Sex. Marital Ther., 1987, 13 : 239253.

18. DAVIES T.F., GOMEZ-PAN A., WATSON M.J., MOUNT JOY C.Q., HANKER J.P., BESSER G.M. et al. : Reduced gonadotrophin response to releasing hormone after chronic administration to impotent men ; Clin. Endocrinol., Oxford, 1977, 6 : 213-218

19. DE LA FUENTE R.B., KROPMAN R.F., MEINHARDT W., LYCKLAMA à NIJEHOLT A.A.B., ZWARTENDIJK J. : Trazodon versus placebo for impotence treatment. First congress of the european society for impotence research (ESIR), Porto Carras, September 1995. Int. J. Impotence Res. 1995, 7, Suppt 1 : 115.

20. DEMYTTENAERE K., VANDERSCHUEREN D. : Selective serotonin reuptake inhibitors and sexual function ; In : The pharmacology of sexual function and dysfunction. J. Bancroft ed. Excerpta medica, Amsterdam, 1995, $6: 327-339$.

21. DORNAN W.A., MALSBURY C.W. : Neuropeptides and male sexual behavior. Neurosci. Biobehav. Rev. $1989,13: 1-15$.

22. EHRENSING R.H., KASTIN A.J., SCHALLY A.V. : Behavioral and hormonal effects of prolonged high doses of LHRH in male impotency. Peptides, 1981, 2 : 115-121.

23. EVANS I.M., DISTILLER L.A. : Effects of luteinizing hormone releasing hormones on sexual arousal in normal men. Arch. Sex. Behav., 1979, 8 : 385-395.
24. EVERITT B.J. : Neuroendocrine mechanisms underlying appetitive and consummatory elements of masculine sexual behaviour ; In : The pharmacology of sexual function and dysfunction. J. Bancroft ed. Excerpta medica, Amsterdam, 1995, 6 : 15-31.

25. EVERITT B.J., BANCROFT J. : Of rats and men : the comparative approach to male sexuality. In : annual review of sex research, J. Bancroft ed., 1991, II, 77-117.

26. EVERITT B.J., HANSEN S. : Catecholamines and hypothalamic mechanisms ; In : Psychopharmacology and Sexual Disorders. D. Wheatley ed. Oxford University Press, 1983, 3-14.

27. FABBRI A., JANNINI E.A., GNESSI L., MORETTI C., ULISSE S., FRANZESE A., et al. : Endorphins in male impotence : evidence for naltrexone stimulation of erectile activity in patient therapy. Psychoneuroendocrinology, 1989, 14 : 103-111.

28. FRAJESE G., LAZZARI R., MAGNANI A., MORETTI C., SFORZA V., NEROZZI D. : Neurotransmitter, opioidergic system, steroid hormone interaction and involvement in the replacement therapy of sexual disorders, J. Steroid Biochem. Molec. Biol. 1990, 37 : 411-419.

29. GAY R.W., DAVIS G.A., BAUM S. : Influences of a specific Dopamine-2 Agonist (L4163502) on erection and sexual performance in primates; Abstracts of the 15th meeting of the international academy for sex research, Princeton, New Jersey, $1989,12$.

30. GIULIANO F., RAMPIN O., BENOIT G., JARDIN A. : Neural control of penile erection ; Urologic clinics of North America, 1995, 22 : 747-766.

31. GLICK B.B., BAUGHMAN W.L., JENSEN J.N., PHOENIX C.H. : Endogenous opiate systems and primate reproduction : inability of naloxone to induce sexual activity in rhesus males. Arch. Sex. Behav.,1982, 11 : 267-276.

32. GOLDSTEIN J.A. : Erectile function and naltrexone. Ann. Intern. Med., 1986, 105 : 799-803.

33. GRUGNI G., CARANI C., MAESTRONI G., GUZZALONI G., ARDIZZI A., LISSONI P. et al. : Melatonin Levels in Psychogenic Impotence ; Horm metab. Res., 1994, 26 : 440-441.

34. HAENSEL S.M., ROWLAND D.L., SLOB A.K. : Serotoninergic drugs and masculine sexual behavior in laboratory rats and men; In : The pharmacology of sexual function and dysfunction. J. Bancroft ed. Excerpta medica, Amsterdam, 1995, 6 : 235-247.

35. HEATON J.P.W., MORALES A., ADAMS M.A. : Recovery of erectile function by the oral administration of apomorphine ; Urology, 1995, 45 : 300306. 
36. HERBERT J. : Neuropeptides, stress and sexuality : Towards a new psychopharmacology ; In The pharmacology of sexual function and dysfunction. J. Bancroft ed. Excerpta medica, Amsterdam, 1995, $6: 77-92$.

37. HOFFMAN B.B., LEFKOWITZ R.J. : Alpha-adrenergic receptors subtypes; N. Engl. J. Med., 1980, $302: 1390-1396$.

38. HUGHES A.M., EVERITT B.J., HERBERT J. : The effects of simultaneous or separate infusions of some pro-opiomelanocortin derived peptides (beta-endorphin, melanocyte stimulating hormone, and corticotropin-like intermediate polypeptide) and their acetylated derivatives upon sexual and ingestive behavior of male rats. Neuroscience, 1989, 27 : 689-711.

39. KENDRICK K.M., DIXSON A.F. : Luteinizing Hormone Releasing Hormone enhances proceptivity in a primate. Neuroendocrinology, 1985, 41 : 449-453.

40. KIM S.C., OH M.M. : Norepinephrine involvement in response to intracorporeal injection of papaverine in psychogenic impotence ; J. Urol., 1992, 147 : 1530-1532.

41. KIMURA Y., KISAKI N., SAKURADA S., TADANO T. : On the brain monoaminergic systems relating to ejaculation. I. Brain dopamine and ejaculation. Andrologia, 1976, $8: 313-320$.

42. KURT U., OXCARDEZ H., ALTUG U. : The efficacy of antiserotoninergic agents in the treatment of erectile dysfunction ; J. Urol., 1992, $152: 407$ 409.

43. LAL S., ACKMAN D., THAVUNDAYIL J.X., KILY M.E., ETIENNE P. : Effect of apomorphine, a dopamine receptor agonist, on penile tumescence in normal subjects ; Progress NeuroPsychopharmacol. Biol. Psychiatry, 1984, 8: 695699.

44. LARSSON K., AHLENIUS S. : Masculine sexual behavior and brain monoamines. In : Psychopharmacology of sexual disorders. Biological Psychiatry - New prospects $\mathrm{n}^{\circ} 4, \mathrm{M}$. Segal ed., J. Libbey, Londres, 1985, 15-32.

45. MAS M., FUMERO B., PEREZ-RODRIGUEZ I., GONZALES-MORA J.L. : The neurochemistry of sexual satiety. An experimental model of inhibited desire; In : The pharmacology of sexual function and dysfunction. J. Bancroft ed. Excerpta medica, Amsterdam, 1995, $6: 115-126$.

46. MORALES A., CONDRA M., OWEN J.A., SURRIDGE D.H., FENEMORE J., HARRIS C. : Is yohimbine effective in the treatment of organic impotence? Results of a controlled trial. J. Urol, 1987, $137:$ 1168-1172.
47. MURPHY M.R. :Endogenous opiates and the mechanism of male sexual behavior. In : Psychopharmacology of sexual disorders. Biological Psychiatry - New prospects $n^{\circ} 4$, M. Segal ed., J. Libbey, Londres, 1985, 51-62.

48. OGAWA S., KUDO S., KITSUNAI Y., FUKUCHI $S$. : Increase in oxytocin secretion at ejaculation in male. Clin. Endocrinol., Oxford, 1980, $13: 95-98$.

49. PARMEGGIANA P.L., MORRISON A.R. : Central regulation of autonomic function; $A D$ Loewy et KM Spyer eds, Oxford University Press, New York, 1990.

50. PFAUS J.G., EVERITT B.J. : The psychopharmacology of sexual behavior ; In : Psychopharmacology, the fourth generation of progress, F.E. Bloom et D.J. Kupfer eds, Raven press, New York, 1994, 743-758.

51. POMERANTZ S.M. : Monoamine influences on male sexual behaviour of non human primates; In : The pharmacology of sexual function and dysfunction. J. Bancroft ed. Excerpta medica, Amsterdam, 1995, 6 : 201-211.

52. POWER-SMITH P. : Beneficial sexual side-effects from fluoxetine ; Br. J. Psychiatry, 1994, 164 : 249250.

53. PRZEWLOCKI R., PRZEWLOCKI B., LASON W. : Stress and beta-endorphin secretions ; In : Neurobiology of opioïds. O.F.X. Almeida et T.S. Shippenberg eds, Springer-Verlag, Berlin, 1991 : 229-243.

54. PSIET J.S., WON LEE S. : The neural mechanism of apomorphin induced erection : an experimental study by comparison with electrostimulation induced erection in the rat model ; J. Urol., 1994, 152 : 2125-2128.

55. REID K., SURRIDGE D.H.C., MORALES A., CONDRA M., HARRIS C., OWEN J. et al. : Double-blind trial of yohimbine in the treatment of psychogenic impotence ; Lancet, 1987, ii, 421423.

56. RILEY A.J. : Oxytocin and coitus. Sex. Marit. Ther., 1988, $3: 29-36$.

57. RILEY A.J. : Alpha adrenoceptors and human sexual function; In: The pharmacology of sexual function and dysfunction. J. Bancroft ed. Excerpta medica, Amsterdam, 1995, $6: 307-322$.

58. RILEY A.J., GOODMAN R.E., KELLER J.M., ORR R. : Double-blind trial of yohimbine hydrochloride in the treatment of erection inadequacy ; Sex. Marit. Therapy., 1989, 4 : 17-26.

59. ROSEN R.C., ASHTON A.K : Prosexual Drugs : Empirical Status fo the "New Aphrodisiacs" ; Arch. Sex. Behav., 1993, $22: 521-543$. 
60. SAENZ DE TEJADA 1., WARE J.C., BLANCO R. : Pathophysiology of prolonged penile erection associated with trazodone use ; J. Urol, 1991, 145 : 6066.

61. SONDA L.P., MAZO R., CHANCELLOR M.B. : The role of yohimbine for the treatment of erectile impotence ; J. Sex. Marital Ther., 1990, 16 : 15-22.

62. SEGRAVES R.T. : Dopamine agonists and their effect on the human penile erectile response ; In : The pharmacology of sexual function and dysfunction. J. Bancroft ed. Excerpta medica, Amsterdam, 1995, $6: 225-229$.

63. STRATTA P., MANCINI F., CUPILLARI M., ROSSI A., CASACCHIA M. : Fluoxetine in premature ejeculation ; Human Psychopharmacology, 1993, $8: 61-64$.

64. SUSSET J.G., TESSIER C.D., WINEZE J., BANSAL S., MALHOTRA C., SCHWACHA M.G. : Effect of yohimbine hydrochloride on erectile impotence : a double-blind study ; J. Urol., 1989, 141 : 1360-1363.
65. TENNENT G., BANCROFT J., CASS J. : The control of deviant sexual behavior by drugs : a double-blind controlled study of benperidol, chlorpromazine and placebo. Arch. Sex. Behav., 1974, $3: 261-271$.

66. TINDAL R.J.S. : Stimuli that cause the release of oxytocin ; In : Handbook of physiology, Endocrinology 4. Geiger ed., American Physiology Association, Washington DC, 1975.

67. WIEDEKING C., ZIEGLER M.G., LAKE C.R. : Plasma noradrenaline and dopamine beta-hydrosylase during human sexual activity ; J. Psychiatr. Res., 1979, $15: 139-145$. 